\title{
Revista de Estudios Sociales
}

\section{La cliometría en Colombia: una revolución interrumpida, 1971-1999}

Adolto Meisel Roca

\section{OpenEdition}

1 Journals

Edición electrónica

URL: https://journals.openedition.org/revestudsoc/28602

ISSN: $1900-5180$

Editor

Universidad de los Andes

Edición impresa

Fecha de publicación: 1 junio 2001

Paginación: 57-64

ISSN: 0123-885X

Referencia electrónica

Adolto Meisel Roca, «La cliometría en Colombia: una revolución interrumpida, 1971-1999», Revista de Estudios Sociales [En línea], 09 | 01/06/2001, Publicado el 19 noviembre 2018, consultado el 04 mayo 2021. URL: http://journals.openedition.org/revestudsoc/28602

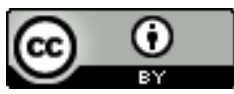

Los contenidos de la Revista de Estudios Sociales están editados bajo la licencia Creative Commons Attribution 4.0 International. 


\section{Lacliometría en Colombia: una revolución interrumpida, 1971-1999.}

\section{Adolíio Meisel Roca}

En 1971 se publicó uno de los libros más controvertidos sobre historia económica de Colombia de las últimas décadas: An Economic History of Colombia, 1845-1930, de William Paul McGreevey'. En ese momento los principales textos de historia económica del país seguían siendo los de Luis Eduardo Nieto Arteta, Economía y cultura en la historia de Colombia, publicado en 1942, y el de Luis Ospina Vásquez, Industria y protección en Colombia, 1810-1930, aparecido en 1955.

La obra de Nieto Arteta puede ser considerada como la pionera de los estudios de historia económica en el país. Se trata de una historia económica de Colombia que abarca desde finales del siglo XVIII hasta finales del XIX. El libro de Nieto contenía algunas innovaciones importantes: se basó en el materialismo histórico, utilizó como fuente de información primaria las Memorias de hacienda, que hasta la fecha no habían sido muy aprovechadas, en algunos capítulos siguió un enfoque regional, muy pertinente en el caso colombiano, y en la mayoría de los capítulos finales el hilo conductor lo constituyó el sector externo. Todas esas características contribuyeron a que entre 1942 y 1971 fuera el texto más leído de historia económica del país.

Sin embargo, el trabajo de Nieto acusa serias deficiencias. El autor era un joven abogado que a los 25 años se embarcó en la tarea de escribir una obra que contribuyera a: "...la definición de una nueva interpretación de los hechos de la historia de Colombia"2. Su formación económica era mínima y por tal motivo sus análisis a menudo se concentran en los aspectos sociales o resultan muy superficiales. Por ejemplo, las abundantes cifras sobre los flujos de importaciones y

\footnotetext{
* Trabajo preparado para la reunión anual de LACEA, Buenos Aires, 22 al 24 de octubre, 1998. El autor agradece los comentarios de Haroldo Calvo Stevenson.

** M.Phil. en sociología de Yale University y Ph.D. en economía de la Universidad de Illinois. Actualmente es el gerente del Banco de la República de Colombia en Cartagena de Indias.

En ese año también se publicó la Introducción a la historia económica de Colombia de Alvaro Tirado Mejía. Esta es una obra de síntesis y popularización escrita desde una perspectiva marxista por uno de los integrantes de la llamada "nueva historia". Una de las principales características del libro es que es completamente descriptivo. Además, aunque es una obra bastante elemental, resume buena parte de la literatura que sobre el tema se había publicado para la época en que se escribió. Todo ello contribuyó para convertirlo en el libro de historia económica de Colombia más exitoso de todos los tiempos, con veinte ediciones y más de 100.000 copias vendidas.

2 Luis Eduardo Nieto Arteta, "Prólogo a la primera edición", en Economía y cultura en la historia de Colombia, Bogotá, 1942.
}

exportaciones que incluye, siempre se presentan en términos nominales, a pesar de que en la última parte del siglo hubo una inflación relativamente sostenida.

En el ámbito de las interpretaciones más generales, Nieto Arteta siguió muy de cerca a los ideólogos liberales colombianos del siglo pasado (como Salvador Camacho Roldán, Anibal Galindo, José María Samper, Rafael Nuñez y Miguel Samper). El libro esta atiborrado de citas extensas de esos autores, lo cual hace que su lectura no sea tarea fácil. En efecto, como lo ha señalado Gonzalo Cataño, el $51 \%$ de la extensión de la obra la constituyen las citas de otros autores y el texto de Nieto alcanza a ser de solo el $49 \% 3$. Peor aún, a menudo utiliza como única evidencia de sus aseveraciones las citas de personajes que fueron actores políticos en muchos de los temas sobre los que escribieron.

Además, el libro en muchas ocasiones presenta simplificaciones excesivas que, aunque tal vez acordes con el paradigma utilizado por el autor, no reflejan la realidad histórica colombiana. Por ejemplo, utilizó una dicotomía según la cual los miembros del Partido Liberal representaban los intereses de los comerciantes y los del Partido Conservador los de los terratenientes ${ }^{4}$. También su análisis de la economía colonial, en la cual contrasta la economía de la región Oriental, que interpreta como anticolonial, manufacturera, comercial y agrícola, y la del Centro, que según Nieto, era colonial y latifundista, deja por fuera a la Costa Caribe, Antioquia y el Cauca, es decir más o menos la mitad del país. Como resultado, por ejemplo, la esclavitud está por fuera de su interpretación de la economía colonial.

Con todas estas limitaciones, resulta un poco sorprendente, desde una perspectiva actual, el enorme impacto de Economía y cultura en la historia de Colombia. Dos factores, al menos, pueden ayudar a explicarlo: el hecho, por una parte, de que su enfoque fuese más o menos marxista, en una época en que esa ideología ganaba adeptos entre los principales intelectuales colombianos, y especialmente entre la generación de historiadores que se formaron en la década de 1960, y, por otra parte, por cuanto no había ninguna otra obra que abarcara la historia económica del país para un período de tiempo más o menos extenso.

\footnotetext{
3 En algunos capítulos el porcentaje del texto constituido por las citas se eleva mucho más. Por ejemplo, en el capítulo XIV constituyen el 79\%. Al respecto, véase Gonzalo Cataño,"Un clásico de la historiografía nacional: Economía y cultura de Luis E. Nieto Arteta", en Historia crítica, No. 15, junio-diciembre, 1997.

4 Al respecto, véase el excelente ensayo de Frank Safford, "Aspectos sociales de la política en la Nueva Granada, 1825-1850", en Frank Safford, Aspectos del siglo XIX en Colombia, Bogotá, Ediciones Hombre Nuevo, 1977.
} 
El libro de Luis Ospina Vásquez, por su parte, cubre un periodo de la historia de Colombia similar al de Nieto Arteta. En casi todo lo demás estos dos trabajos son completamente distintos (al igual que sus autores) ${ }^{5}$. Mientras el libro de Nieto Arteta es una obra de juventud, el de Ospina Vásquez pertenece a la madurez intelectual de su autor. Además, Ospina tuvo entrenamiento en economía (posgrado en el London School of Economics) y realizó una investigación a lo largo de varios años, incluso consultando el Archivo Nacional, para preparar este trabajo. No intentó escribir una historia económica del período sino, más bien, quiso estudiar la evolución de la industria nacional entre 1810 y 1930 y las políticas económicas que la afectaron, en especial las de comercio exterior.

Se trata de una muy sólida obra de historia económica institucional tradicional. Como tal, el autor va describiendo por períodos el desarrollo de las instituciones y los eventos relacionados con la industria. Tiene muy pocas cifras, que se van tejiendo con la narrativa, y casi ninguna serie. En contraste con Nieto Arteta, tampoco hay un marco teórico explícito desde el cual el autor orienta su investigación. En gran medida por ello, y por el talante intelectual del autor, que hace un gran esfuerzo por fundamentar con evidencia empírica sus aseveraciones, hay muy pocas generalizaciones e interpretaciones globales en esta obra. Como consecuencia, casi toda la obra de Ospina Vásquez tiene validez actual pues sólo rara vez sus argumentos estaban claramente equivocados ${ }^{6}$.

Una de las mayores limitaciones del libro de Ospina Vásquez es que, al no contar con una base estadística amplia, su análisis de la evolución económica global es totalmente "impresionista", aunque revela una sólida noción de las magnitudes económicas ${ }^{7}$. A pesar de ello, no superó a Nieto Arteta en la utilización de cifras nominales.

\footnotetext{
5 Ospina Vásquez era hijo del presidente Pedro Nel Ospina y por el lado materno provenía de una de las familias de empresarios más importantes de Antioquia. En contraste, Nieto Arteta provenía de la clase media del Caribe Colombiano.

6 Uno de esos pocos casos es su aceptación del argumento de que la devaluación nominal del peso a finales del siglo XIX estimuló las exportaciones de café. Este argumento lo esgrimieron inicialmente los miembros del gobierno y posteriormente lo retomaron numerosos historiadores colombianos. Sin embargo, al calcular la tasa de cambio real se observa que la devaluación fue sólo nominal. Al respecto, véase Adolfo Meisel Roca y Alejandro López Mejía, "Papel moneda, tasas de interés y revaluación durante la Regeneración", en El Banco de la Republica, Antecedentes, evolución y estructura, Bogotá, Banco de la Republica, 1990.

7 En Colombia los primeros cálculos del Producto Interno Bruto se hicieron a fines de la década de 1940. Por varias de las observaciones que hace, Ospina Vásquez parece haber estado familiarizado con estas estimaciones.
}

El estilo de exposición de Ospina Vásquez y las demás características de la obra lo convirtieron en "un historiador de historiadores", pero su popularidad en círculos más amplios fue limitada. Por tal motivo, mientras el libro de Nieto ha tenido hasta la fecha veinte ediciones, el de Ospina solo lleva cinco. Además, su segunda edición solo apareció en 1974, casi veinte años después de la primera.

En 1971 se publicó en inglés una obra que estaba destinada a generar la mayor controversia que se ha dado en torno a una obra sobre historia económica en Colombia. Me refiero a la obra del entonces joven economista estadounidense William Paul McGreevey, An Economic History of Colombia, 1845-1930. La obra estaba basada en la investigación que su autor realizó para su tesis de Ph.D. en economía en MIT y que presentó en 1965. El autor estaba inmerso en la literatura de la revolución cliométrica que por ese entonces se imponía en los departamentos de economía de los Estados Unidos ${ }^{8}$. En un seminario que se realizó en Bogotá en 1975 sobre su libro, le escuche decir a McGreevey que cuando decidió hacer su tesis sobre Colombia, las dos cosas que sabía sobre el país eran que producía café y que la capital era Bogotá. Además, señaló que había escogido a este país pues, al ir a la biblioteca, constató que no había una sola obra general sobre el desarrollo económico colombiano. Su intención era inaugurar en nuestro medio la triunfante revolución cliométrica. En el prólogo a la primera edición en español de la obra, el traductor, el economista Haroldo Calvo Stevenson, señaló que: "...el trabajo de McGreevey constituye el debut de la nueva historia económica en la historiografía del caso colombiano"9. Desde el momento mismo en que se publicó el libro tuvo un enorme impacto entre los economistas. En 1973 yo era estudiante del primer año de economía en la Universidad de los Andes y recuerdo muy bien la avidez y el enorme entusiasmo con la cual mis condiscípulos más avanzados leían a McGreevey.

El libro está estructurado en torno a tres secciones. En la primera el autor analiza los antecedentes, un poco como lo hicieron Nieto Arteta y Ospina Vásquez, pero ampliándolos hasta 1845, para resaltar la continuidad entre la colonia y las primeras décadas de la república (en esta interpretación sigue

\footnotetext{
8 Claudia Goldin, "Cliometrics and the Nobel", en Journal of Economic Perspectives, Vol. 9, No. 2, Spring, 1995, pág.193. Por cliometría se entiende el estudio de la historia económica por medio de la aplicación de la teoría económica y los métodos estadísticos.

9 Haroldo Calvo Stevenson, "Prólogo del traductor", en William Paul McGreevey, Historia económica de Colombia, 1845-1930, Bogotá, Ediciones Tercer Mundo, 1975.
} 
a Nieto Arteta). Del análisis de esta primera parte tal vez lo que más llama la atención es su interpretación de los flujos de comercio y de capitales hacia España, sobre la base de una información muy dispersa y poco confiable. A mi entender, la utilización de las cifras en estos capítulos en nada se parece a la propuesta de la Nueva Historia Económica sobre la utilización de métodos cuantitativos en forma rigurosa. Tampoco se parece al uso de las cifras por parte de los historiadores económicos tradicionales, que tiende a ser para ilustrar aspectos específicos de la narración. McGreevey, haciendo gala de sus conocimientos de economía internacional y con unos precarios conocimientos sobre la economía colonial del Virreinato de la Nueva Granada, se lanzó a una interpretación del funcionamiento del sector externo neo-granadino que, aunque consistente lógicamente, partía de supuestos equivocados. Como veremos más adelante, Frank Safford explicó con contundencia las fallas en la argumentación de McGreevey en el seminario que se realizó en 1975 en Bogotá para discutir su obra ${ }^{10}$.

La siguiente sección del libro analiza el período 18451885. Tal vez lo más innovador aquí es la utilización de fuentes extranjeras para construir las series de comercio exterior (Inglaterra, Francia, Estados Unidos, Hamburgo, Bremen). McGreevey utilizó estas fuentes porque en esa época había la idea de que las cifras del comercio exterior colombiano en el siglo XIX eran muy poco confiables debido al contrabando, en el caso de las exportaciones, y a la subfacturación, en el caso de las importaciones. Aunque la innovación era muy prometedora, la forma en que realizó los cálculos está plagada de ajustes ad hoc, que además no explica en forma detallada, razón por la cual sus datos resultan también poco confiables. Al respecto, autores como Alberto Umaña han señalado que las cifras de Estados Unidos e Inglaterra también tienen problemas por cuanto se presentaban fenómenos que sesgaban los resultados, como la re-exportación a través de Panamá, perteneciente por esa época a Colombia ${ }^{11}$.

La última parte del libro contiene, a mi juicio, uno de los análisis más débiles tanto analítica como empíricamente y, simultáneamente, el aporte principal del autor al conocimiento de la historia económica de Colombia, que infortunadamente, hasta ahora ha pasado casi

10 Instituto de Estudios Colombianos, Historia de Colombia, Un debate en marcha, Bogotá, Biblioteca Banco Popular, 1979.

11 Alberto Umaña, "Problemas estadísticos en el análisis del periodo liberal, 18451885", en Instituto de Estudios Colombianos, Historia de Colombia, Un debate en marcha, Bogotá, 1979. completamente desapercibido entre los historiadores económicos colombianos.

Me quiero referir ahora al aspecto de la tercera sección del libro de McGreevey que más ha generado controversia: su teoría de por qué Colombia vivió una transición hacia el crecimiento entre finales del siglo XIX y comienzos del presente. El análisis está escrito en la jerga de la teoría neoclásica del crecimiento, pero es de un simplismo sorprendente. El autor arguye que, en algún momento después de 1850, ocurrió un desplazamiento autónomo del potencial de crecimiento de la población colombiana. Ese aumento demográfico:

...incrementó la interdependencia entre personas y regiones $y$, por consiguiente, generó nuevos problemas de adaptación y nuevas oportunidades para introducir innovaciones que redujeran los costos, particularmente en lo que se refiere a las mejoras en el transporte y a la urbanización... Algunas regiones aprovecharon al máximo las oportunidades existentes, mientras que otras parecen haberse visto abrumadas por el problema del crecimiento demográfico y, por lo tanto, haberse estancado. En última instancia, la explicación forzosamente radica en la motivación humana: algunas regiones se desarrollaron porque sus gentes así lo desearon ${ }^{12}$.

Independientemente de la validez teórica de su argumentación, no existe evidencia alguna de que en la segunda mitad del siglo XIX hubiera ocurrido un desplazamiento de la tasa de crecimiento potencial de la población colombiana. Lo que indican las cifras censales es que ese crecimiento fue más o menos estable a lo largo de todo el siglo. Lo que sí hubo-y McGreevey no analizafueron claras diferencias inter-regionales en esas tasas de crecimiento. Peor aún, el autor no hace ningún intento de demostrar que las regiones que se rezagaron "no desearon desarrollarse" y viceversa.

En mi concepto, el mejor capítulo del libro de McGreevey es el penúltimo, debido a que realiza una evaluación de costobeneficio de la construcción de los ferrocarriles en la década de 1920, tal vez la década de mayor inversión en infraestructura de transporte en la historia del país. Esas inversiones fueron duramente criticadas por muchos observadores de la época, por considerar que hubo mucha

\footnotetext{
12 William Paul McGreevey, Historia económica de Colombia, 1845-1930, Bogotá, Tercer Mundo Editores, 1982, pág. 287.
} 
corrupción y que fueron muy ineficientes. Por esa razón, acuñaron la frase la "danza de los millones" para referirse a esas inversiones. Varios historiadores económicos han aceptado, sin muchos elementos de juicio, las aseveraciones de esos críticos. Sin embargo, McGreevey encontró que, en general, para el periodo 1920-1949 los ferrocarriles colombianos fueron una inversión rentable ${ }^{13}$. Tanto por el manejo de cifras, como por la explicación clara de la metodología utilizada, la evaluación de costo-beneficio de las inversiones en ferrocarriles que realizó McGreevey son bastante convincentes. Es esta, a mi juicio, la principal revisión que hizo el autor a la historiografía económica colombiana.

En 1975, un grupo de historiadores colombianos comandados por Marco Palacios organizó un seminario en Bogotá para discutir durante dos días la obra de McGreevey ${ }^{14}$. Allí se congregaron casi todos los principales historiadores colombianos exponentes de la llamada "nueva historia", que se habían formado en la década de 1960 bajo la orientación de Jaime Jaramillo Uribe y con una fuerte influencia del marxismo y de la Escuela de los Annales ${ }^{15}$. Además, participó Luis Ospina Vásquez, en ese momento el historiador económico colombiano con más prestigio. También estuvieron presentes los mas destacados historiadores colombianistas de Estados Unidos y Europa: Frank Safford, Malcolm Deas, David Bushnell, J.Leon Helguera y Christopher Abel. Todos los expositores fueron críticos del trabajo de McGreevey o de algún aspecto del mismo. Ninguno encontró que el trabajo contribuía al conocimiento de la historia económica del país.

El seminario se inició con el más demoledor de todos los trabajos presentados, el de Frank Safford, paradójicamente un compatriota del autor de esta controvertida obra. No hay en toda la producción historiografica colombiana una pieza tan critica de un trabajo académico como esta de Safford. Su escrito es algo casi único en la historiografía de un país donde hay una muy débil tradición de crítica seria sobre las obras históricas.

${ }^{13}$ En efecto, McGreevey encontró una tasa de ahorro social tan alta como el estimativo máximo de Fogel para los ferrocarriles de Estados Unidos en 1890, McGreevey, Historia económica de Colombia..., pág. 273.

${ }^{14}$ Cabe señalar que en 1979 Marco Palacios publicó el estudio histórico mas importante sobre el café que se ha hecho en el país, El café en Colombia, una historia económica, social y política, Bogotá, Editorial Presencia, 1979. En este ensayo no hemos incluido esta excelente obra debido a que tiene objetivos mas amplios, lo cual es una de sus fortalezas, por cuanto el autor estudió también los aspectos empresariales, políticos y sociales, razón por la cual solo unos capítulos tienen énfasis en los aspectos económicos.

15 De los principales exponentes de la "nueva historia" sólo estuvieron ausentes Germán Colmenares y Jorge Orlando Melo.
La evaluación que hizo Safford fue totalmente negativa:

muchas ideas no siempre están bien consideradas y a veces parecen contradictorias; además el libro, como obra histórica, sufre de muchos errores y como análisis económico de varios lapsus notables...Aunque hay muchos errores factuales en la obra no voy a intentar anotar todos estos por ser una tarea muy larga y abrumadora. Espero, sin embargo, por medio del comentario sobre método y análisis, ayudar a crear una base para juzgar el libro con ojos prevenidos ${ }^{16}$.

Y lo logró... y de que manera ${ }^{17}$.

Cabe aclarar que Frank Safford nunca atacó directamente a la Nueva Historia Económica en su discusión del libro de McGreevey. Se concentró en poner en evidencia la forma poco rigurosa como éste manejó la evidencia empírica y en mostrar algunos de los principales problemas de análisis del trabajo. Incluso, Safford señaló que esas deficiencias no eran atribuibles a los métodos de la NHE:

El libro carga mucha estadística ficticia y alegre, como también análisis fallidos. Él justifica la estadística inventada como ejemplo de la nueva historia económica, llamada la historia contrafactual. En realidad, viola, o, mejor dicho, a veces no hace

${ }^{16}$ Frank Safford, "Algunos problemas de método y análisis del libro de William Paul McGreevey", en Instituto de Estudios Colombianos, Historia de Colombia, pág. 25.

17 Aparentemente una razón por la cual Safford fue tan vehemente en sus críticas a McGreevey es que sentía que ese autor no le había dado crédito por la utilización de muchas de las cifras que el había recopilado en sus trabajos. Yo considero que tal vez había algo más de fondo. Por esa época en los Estados Unidos la triunfante revolución cliométrica desplazaba a los historiadores económicos tradicionales de los departamentos de economía. Como en toda revolución, seguramente se cometieron excesos con la generación anterior de historiadores económicos que eran o historiadores con poco entrenamiento en economía o economistas con una formación matemática y estadística limitada. Por todo ello, en los departamentos de historia de los Estados Unidos los cliometristas eran vistos "con ojos prevenidos". Refiriéndose a la actitud de los historiadores económicos tradicionales (fueran economistas o historiadores) hacia la NHE a comienzos de la década de 1970, David Landes señaló que: "...aunque no rechazaban la medición y el análisis cuantitativo directamente, como lo hicieron muchos de los historiadores no-económicos... argumentaban que la nueva cliometría era una novedad no-científica en sus métodos, con fallas en su lógica, limitada en su aplicabilidad por las limitaciones de la información histórica y por la complejidad y la ambigüedad del cambio histórico. Pero ellos decían todo esto más en la privacidad de sus hogares y salones de clase que en escritos públicos, debido a que (ahora se puede decir) la mayoría estaban demasiado intimidados para retar a la Nueva Historia Económica al combate. No podían entender una parte de los nuevos trabajos. A veces no podían ni leerlos". David Landes, "On Avoiding Babel", en Journal of Economic History, Vol. 38, 1978, pág. 6. 
caso de los pocos cánones que tienen los nuevos historiadores económicos, y así da una mala impresión de lo que se puede hacer con esta técnica ${ }^{18}$.

\section{A diferencia de Safford, Marco Palacios orientó una} parte de su critica a los métodos de la NHE. Por ejemplo, puso en duda que la historia se pueda escribir a través de la construcción de modelos matemáticos de la cual se derivan unas hipótesis que luego se aceptan o rechazan de acuerdo con la evidencia empírica disponible, que es la forma como Palacios concibe los métodos de la NHE. Por ello afirma: "El principal objetivo de este trabajo es argumentar contra tales métodos" ${ }^{\prime 19}$. En general sus objeciones contra el uso de modelos teóricos son poco convincentes ya que se limita a traer a colación las ideas del historiador marxista Witold Kula, en el sentido de que se debe tener en cuenta el modo de producción existente, y a señalar que las variables dependientes se pueden volver independientes y viceversa, es decir que puede haber problemas de simultaneidad entre las variables.

Es necesario resaltar que en el seminario en mención nunca se hizo claridad que lo que allí se estaba refutando no eran los métodos de la NHE, sino la forma como un economista joven y poco conocedor de Colombia trató, sin mucho éxito, de estudiar la historia económica nacional aplicando esos métodos. Por esa razón, entre muchos de los asistentes a este simposio, así como entre quienes luego leyeron la publicación de los trabajos presentados, quedó la idea de que la Nueva Historia Económica tenía serias limitaciones metodológicas y que enfoques alternativos como el marxismo, los Annales o la historia social anglo-sajona de corte empirista eran mucho mas útiles $^{20}$.

Habría que preguntarse por qué, en un medio donde es completamente inusual que un trabajo no sólo histórico sino intelectual se someta a una crítica tan sistemática, se realizó este

\footnotetext{
18 Instituto de Estudios Colombianos, Historia de Colombia, pág. 26.

${ }^{19}$ Marco Palacios, "Las condiciones de oferta de café. Una aproximación de crítica socio-histórica al modelo empleado por McGreevey", Ibid., pág. 171.

20 En 1975 yo estudiaba tercer año de economía y con otros estudiantes de la Universidad de los Andes asistí al seminario sobre el libro de McGreevey. Lo que escuché allí me convenció de que la NHE no era útil para los estudios de historia económica de Colombia y que sus métodos eran muy cuestionables. Además, quedé con la idea de que era principalmente a los autores marxistas como Witold Kula, profusamente referenciado en el trabajo de Marco Palacios, a quienes había que estudiar, lo cual hice en los siguientes años, sin preocuparme entonces por conocer a los principales exponentes de la NHE, como Fogel, North y Engerman.
}

simposio sobre el libro de McGreevey²1. La explicación que dieron los organizadores del evento fue que era la primera vez que aparecía una obra con un título tan ambicioso como Historia económica de Colombia, 1845-1930. Es obvio que esa no es la explicación. Más bien habría que recordar que, con el libro de McGreevey, hizo su debut en Colombia la Nueva Historia Económica. Los economistas y los estudiantes de economía estaban leyendo con entusiasmo el libro. Por ello un grupo de historiadores colombianos formados bajo las influencias de Luis Ospina Vásquez, el marxismo, la dependencia, la escuela francesa de los Annales y la historia social anglosajona, intentó frenar la difusión en nuestro país de las ideas de la NHE. Y lo lograron, pero por las razones equivocadas. El libro de McGreevey recibió críticas por dos tipos de razones: de una parte, el manejo de las cifras y los análisis que realizó el autor y, de otra, la utilización de los métodos de la NHE. Pues bien, las críticas a los métodos de la Nueva Historia Económica fueron bastante superficiales. En contraste, las críticas al manejo de las cifras y a los análisis del autor fueron demoledores. Al no hacerse claridad sobre esto, por cuanto todos los expositores escogidos estaban en contra de la Nueva Historia Económica, quedó la idea equivocada de que en la Sabana de Bogotá se había demostrado que este enfoque tenía serias limitaciones.

Hemos dedicado bastante atención a la recepción en Colombia del libro de McGreevey por cuanto consideramos que ayuda a entender por qué en los siguientes veinte años, por lo menos, los estudios de historia económica en Colombia estuvieron dominados por el marxismo, los Annales, la dependencia, y hubo una ausencia completa de la NHE. Es cierto que lo mismo sucedía en toda América Latina, como lo ha señalado recientemente Stephen Haber ${ }^{22}$. Sin embargo, el colombiano parece haber sido un caso extremo de esa tendencia, a mi juicio como consecuencia del desastroso debut de la NHE en nuestro medio y de la forma en que los críticos de McGreevey lograron que se asociaran los defectos de la obra de ese autor con la cliometría.

${ }^{21}$ Incluso en la presentación del libro se hace referencia a lo singular de este seminario: "A muchos pareció y sigue pareciéndoles inusitada la idea del I.E.C. de realizar un seminario de historia económica de Colombia alrededor de un libro escrito por un norteamericano. Para otros, es un despropósito. Podría discutirse ad infinitum, pero el tema no lo merece. Si en Colombia no se organizó nunca un seminario sobre El Quijote o sobre Cien años de soledad, la supuesta responsabilidad recaería en los hombres dedicados a la literatura", en "Presentación", Instituto de Estudios Colombianos, Historia de Colombia, pág. 9.

22 Stephen Haber, "Introduction: Economic Growth and Latin American Economic Historiography", en Stephen Haber, editor, How Latin America Fell Behind, Essays on the Economic Histories of Brazil and Mexico, 1800-1914, Stanford University Press, 1997, pág. 7. 
En 1973, se publicó el primer tomo de la Historia económica y social de Colombia, 1537-1719, de Germán Colmenares. El segundo tomo, con el subtítulo de Popayán : una sociedad esclavista, 1680-1800, salió al mercado en 1979. Todavía hoy, veinticinco años después, este trabajo sigue siendo la principal obra sobre la historia económica y social de Colombia en el periodo colonial.

Colmenares había sido alumno de Jaime Jaramillo Uribe, quien es considerado, con Luis Eduardo Nieto Arteta y Luis Ospina Vásquez, uno de los tres pioneros de la llamada Nueva Historia que en la década de 1960 renovó la historiografía colombiana. Hasta ese momento los estudios históricos en el país estaban dominados por las Academias de Historia y por un enfoque narrativo de "grandes hombres" en la guerra y en la política ${ }^{23}$. Este primer tomo de la obra de Colmenares estaba basado en su tesis de doctorado en la Universidad de París, donde recibió la influencia directa de la Escuela de los Annales a través de Fernand Braudel y Pierre Vilar (miembro este último de su comité de tesis) ${ }^{24}$.

El libro esta fundamentado en una extensa investigación en los archivos de Colombia y en el Archivo General de Indias en Sevilla. Desde el punto de vista de la historia económica, los aportes más importantes de la obra se hallan en el capítulo sobre la minería de oro, el principal y casi único producto de exportación de Colombia durante el período colonial. Entre otros aportes, Colmenares construyó la serie de la producción aurífera entre 1541 y 1660.

En el segundo tomo, Colmenares estudió la sociedad esclavista de la provincia de Popayán en el siglo XVIII. El énfasis recayó en la historia social de la esclavitud más que en sus aspectos económicos. Por lo tanto, examina los distintos aspectos demográficos de la trata de esclavos y de las estructuras de edades de las poblaciones esclavas. Aunque el autor conocía los principales trabajos de la NHE sobre la esclavitud, expresó algunas dudas sobre su validez y no intentó hacer el tipo de análisis económico y estadístico riguroso que Fogel y Engerman realizaron para la esclavitud en el Sur de los Estados Unidos ${ }^{25}$.

Tal vez el libro de historia económica más influyente del periodo que estamos analizando ha sido el de José Antonio

\footnotetext{
23 Sobre los aportes de Jaime Jaramillo Uribe al desarrollo de los estudios históricos en Colombia, véase: Jaime Jaramillo Uribe, Vida y obra, Bogotá, Archivo General de la Nación, 1996.

24 Germán Colmenares, Historia económica y social de Colombia, 1537-1719, Universidad del Valle, 1973, pág. II.

25 Robert William Fogel y Stanley L. Engerman, Time on the Cross, The Economics of American Negro Slavery, W.W. Norton and Company, 1995.
}

Ocampo, Colombia y la economía mundial, 1830-1910, publicado en 1984. El autor se inspiró en el dependentismo latinoamericano para estudiar la economía colombiana del siglo pasado en función de su articulación al complejo del capitalismo mundial ${ }^{26}$.

Ocampo realizó un análisis muy riguroso del comercio exterior colombiano del siglo XIX, a partir de las cifras de los anuarios estadísticos publicados por el gobierno nacional $y_{\text {, }}$ especialmente, de las memorias de los ministros de hacienda. La obra está lejos de las versiones simplistas del dependentismo latinoamericano. Su aporte principal es haber llevado a cabo un trabajo empírico sólido para construir las series de las exportaciones e importaciones totales, desagregadas por productos y países. Paradójicamente, las cifras que utiliza Ocampo son casi idénticas a las que manejó Luis Eduardo Nieto Arteta. Sin embargo, como se menciono anteriormente, este último nunca superó el análisis de las cifras nominales. Por el contrario, el gran avance metodológico de Ocampo es que construye las series en términos reales per cápita, lo cual le permite trazar las tendencias y fases del comercio exterior entre 1830 y 1930. Curiosamente, las fases que encontró Ocampo en la evolución del comercio exterior en el siglo pasado son casi idénticas a las que halló el criticado McGreevey.

En 1987, José Antonio Ocampo editó el libro Historia económica de Colombia, que se ha convertido, conjuntamente con el de Tirado, en el texto introductorio mas leído sobre el tema. En cierta forma las tendencias dominantes en los estudios sobre historia económica de Colombia hacia fines de la década de 1980 se manifiestan claramente en este trabajo. La obra está organizada cronológicamente en siete capítulos escritos por seis autores principales. Sólo dos de esos autores eran economistas; los otros cuatro eran historiadores. Tres de esos historiadores tenían influencias del marxismo, la Escuela de los Annales, o de ambos. El otro historiador, Jaime Jaramillo Uribe, tenía influencia tanto de Max Weber, como de la historia social anglo-sajona. Los economistas del grupo, Jesús Antonio Bejarano y José Antonio Ocampo, se habían iniciado en sus escritos sobre historia económica de Colombia como seguidores del marxismo y del dependentismo latinoamericano, respectivamente. Por ningún lado apareció en este libro la NHE, completamente desprestigiada después del descalabro que sufrió con el libro de McGreevey.

\footnotetext{
${ }^{26}$ José Antonio Ocampo dice en la introducción: "...creemos identificarnos con los postulados básicos del dependentismo latinoamericano", José Antonio Ocampo, Colombia y la economía mundial, 1830-1910, Bogotá, Siglo Veintiuno Editores, 1984, pág. 24.
} 
En mi concepto, lo que veremos en Colombia en los próximos años en los estudios de historia económica es una creciente influencia de la NHE. Varios factores contribuirán a ello. Stephen Haber señala tres causas principales para el retraso en la difusión de la NHE en América Latina: el alto costo de oportunidad de los economistas con formación avanzada, los altos costos para procesar información cuantitativa y la competencia y hegemonía de paradigmas alternativos ${ }^{27}$. Aunque el primer punto seguirá dificultando la difusión de la NHE en nuestro medio, los otros dos han perdido vigencia. Además, hay un factor positivo adicional: en la actualidad en el país hay un número relativamente grande de economistas con Ph.D. de universidades de Estados Unidos e Inglaterra, algo que no sucedía en la década de 1970, por ejemplo. Ese grupo seguramente contribuirá a la mayor presencia de los trabajos de la NHE, tanto por el lado de la demanda como por el lado de la oferta ${ }^{28}$.

Para mí, el libro que augura lo que serán los trabajos sobre historia económica de Colombia en el futuro próximo es el que editó Fabio Sánchez en 1994 sobre historia monetaria y bancaria del país ${ }^{29}$. De los siete capítulos del libro todos fueron escritos por economistas con excepción de uno sobre historia bancaria escrito conjuntamente por un historiador y un economista. Además, los tres capítulos sobre historia monetaria, que ocupan dos terceras partes de la extensión del libro, son claramente ensayos de cliometría, pues aplican teoría económica y econometría en su análisis de los problemas del pasado. Podríamos incluso decir que este libro constituye el retorno de la NHE a Colombia, tras un cuarto de siglo de hibernación.

En un polémico balance sobre situación actual de los estudios sobre historia en Colombia, Jesús Antonio Bejarano argumenta que hacia finales de la década de 1980 en el país

27 Haber, "Introduction: Economic Growth...", págs. 6-7.

28 En la década de 1990 varios de los economistas colombianos que se especializaron en el exterior hicieron tesis de Ph.D. sobre la historia económica nacional: Juan Luis Londoño, "Income Distribution During the Structural Transformation", Harvard University, 1990; Lina Echeverri, Free Banking in Colombia, 1865-1886, Universidad de Georgia, 1991; Fabio Sanchez, The Monetary History of Colombia During the Intenwar Period, 1920-1939, Universidad de Rutgers, 1993; Mauricio Avella, Essays on Public Debt in Historical Perspective. The Colombian Experience, Universidad de Glasgow, 1994; María Teresa Ramirez, On Infrastructure and Economic Growth, Universidad de Illinois, 1998. Además, en la actualidad el economista Marcelo Buchelli prepara una tesis en la Universidad de Stanford sobre la historia económica de la Zona Bananera de Santa Marta.

29 Fabio Sánchez, compilador, Ensayos de historia monetaria y bancaria de Colombia, Bogotá, Tercer Mundo Editores, 1994. se estancaron las investigaciones sobre historia económica ${ }^{30}$. El ensayo de Bejarano tiene un objetivo más amplio que el de hacer un balance sobre la situación de los estudios de historia económica, pues su discusión se enmarca dentro del análisis del auge de la historia cultural y los enfoques posmodernos. Sin embargo, en este artículo sólo me he referido al caso de la historia económica y sólo haré alusión al escrito de Bejarano en este respecto.

Bejarano señala, a mi juicio correctamente, que en contraste con las décadas de 1960 y 1970 en la actualidad los historiadores colombianos han perdido interés en la historia económica. Sin embargo, pienso que Bejarano se equivoca cuando argumenta que en el ámbito internacional la NHE ha perdido vitalidad. En su concepto, los mayores aportes de la NHE se hicieron en la década de 1960 y en particular en los trabajos sobre la esclavitud y los ferrocarriles. Su opinión en este respecto parece ser producto de su falta de familiaridad con la literatura más reciente en este campo. Por ejemplo, en su ensayo no hace mención a un sólo artículo aparecido en algunas de las principales revistas donde publican los cliometristas como: Economic History Review, Explorations in Economic History, Journal of European Economic History, Revista de Historia Economica. Aunque sí menciona varios artículos publicados en el Journal of Economic History, el más reciente es de 1985. Por lo tanto, no debe sorprendernos que Bejarano piense que después de la década de 1970 los cliometristas: "...no han aportado nada nuevo."

En contraste con la opinión de Bejarano en este respecto, y para sólo referirnos al caso de dos de los más destacados exponentes de la NHE, la historiadora económica Claudia Goldin considera que: "Los últimos aportes en la obra de North y Fogel es probable que terminen siendo considerados sus mejores logros" ${ }^{\prime 31}$. Se refiere Claudia Goldin a los trabajos de Fogel en el campo de la antropometría y a los de North sobre las instituciones y su papel en el crecimiento económico ${ }^{32}$.

Creo que Bejarano tampoco acierta cuando habla de un notable declive de los estudios sobre historia económica de Colombia durante la década de 1990. Si bien es cierto que los

\footnotetext{
30 Jesús Antonio Bejarano, "Guía para perplejos: Una mirada a la historiografía colombiana", Anuario colombiano de historia social y de la cultura, No. 24, 1997.

31 Claudia Goldin, "Cliometrics and the Nobel", en Journal of Economic Perspectives, Vol. 9, No. 2, 1995, pág. 201.

32 Sobre los estudios de Robert Fogel en antropometría, es decir la relación entre estatura y los niveles de nutrición y salud, véase: Barry Eichengreen, "The Contributions of Robert W. Fogel to Economics and Economic History", en Scandinavian Journal of Economics, No. 96, V. 2, 1994, págs. 175-177.
} 
historiadores han perdido interés por este campo, hay un grupo creciente de economistas, y de algunos pocos historiadores, que viene realizando aportes significativos en áreas como: historia monetaria, historia fiscal, historia económica regional e historia bancaria, entre otras. Además, en la actualidad hay un gran interés por la historia económica del país en el siglo XX. En este sentido, cabe destacar que varios de los más destacados economistas colombianos están elaborando en la actualidad estudios sobre distintos aspectos de la historia económica del país en el siglo actual (Salomón Kalmanovitz, Roberto Junguito, José Antonio Ocampo y Miguel Urrutia, entre otros) ${ }^{33}$. También hemos mencionado la elaboración durante la década de 1990 de un buen numero de tesis de Ph.D. sobre historia económica de Colombia. Una situación muy lejos del "fin de la historia económica" en nuestro medio que proclama Bejarano. 